\title{
Cloud Integrated Low Cost Customizable Smart Medical Chair for Diagnosis and Doctor Assistance
}

\author{
Yokesh Babu Sundaresan ${ }^{1 *}$, Kumaresan $\mathrm{P}^{2}$, Arjun Rajshekhar ${ }^{3}$, \\ Swayamdeepta Sanyal ${ }^{4}$ and Rohan Puri ${ }^{5}$ \\ ${ }^{1-5}$ Third year Computer Science and Engineering and Professors - SCSE, SITE, VIT University, \\ Vellore, TN, India, Pincode 632014 \\ * Corresponding author’s Email: yokeshbabu.s@vit.ac.in
}

\begin{abstract}
The application of embedded systems in the field of Biomedical Sciences provides the means to build an accurate health and vital signs monitor. The usage of superficial and non-superficial sensors allows the extraction of various parameters required for the diagnostics of a patient. Such health monitoring devices are not a viable option for the less fortunate who continue to use traditional detection techniques. Furthermore, the systems commercially produced are not open to customization and hence may not be incremented upon to detect new parameters, hence requiring the purchase of a new system. The proposed system instead grants more customizability and portability to the user to allow the creation of a general purpose health monitor that is offered at a lower cost. In this project, we measure the viability of the proposed system by fabricating sensors of various vital signs such as sweat levels, body temperature, pulse rate and blood oxygenation levels using superficial sensors and a tracking system on a smart phone device that serves as a back end. An alternate cloud model approach has also been proposed considering the benefits of cloud computing and to enact the requirement of the ubiquitous nature of the project.
\end{abstract}

Keywords: Vital Signs Monitor, Medical Frame, Electronic Medical Assistance, Oximeter, Galvanic Skin Response, Pulse Rate Monitor, Electrocardiograph Monitor, Body Temperature Monitoring, Smart Data Acquisition, Cloud Computing

\section{Introduction}

Health and vital signs monitors developed for commercial purposes are sold at reasonable prices that cater to the health professionals who wish to asses a patient's vital signs. But an alternative is required for those who are less fortunate and those who do not have the means to access such medical facilities on demand. Furthermore, the demand of every country to possess an excellent health care system directly implies the necessity in treating patients as quickly and efficiently as possible to prevent a back log of medical care. This has been countered through the ages by the increasing number of doctors to treat the in- creasing number of patients. But for all efforts maintained, cities and countries with high population densities still see delayed doctor appointments. The proposed method assumes to counter these issues by the automation of a part of the diagnostics process. In addition, with the revelations achieved thus far in internet related technologies, cloud computing has become a common place of application based services. Such services also extend to the domain of medical servicing, an example of which can be noted in the Cloud Sensor Sock [1]. Hence a cloud based model has been considered in the proposed method to improve the scope of access of information related to di- 


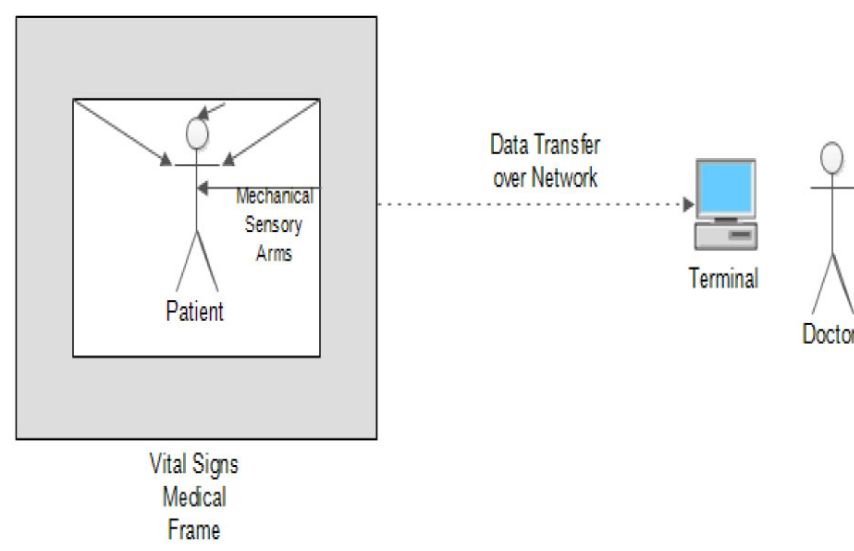

Figure 1 Illustration of medical frame

agnostics.

The prime sector of application of the proposed project lies within the Health Monitoring domain. While a lot of work has already been carried out in this field in the past, a new innovation is required to counter the primary challenges that have been identified in the stated domain, which is listed thusly:

1. Cost effectiveness

2. Portability

3. Ubiquity of information

\section{Customizability}

A prime example of customizability is the concept of a medical frame as shown in Figure 1 which records the vital signs of the patients as they walk through it.

\section{Related Work}

Due to the extensive list of papers that already exist in the field of the Application of Biomedical Science with Embedded Systems, five base papers have been selected as a source of reference of the method proposed. The first base paper [1] implements a Cloud Sensor Sock which relays sensor information to the cloud, namely using the Google App Engine. The Cloud Sensor Sock allows a wearable sensing platform by obtaining information from a heartbeat strap which is connected to an Arduino Lily Pad and then communicated to both an Android device through Bluetooth and directly to the App Engine via the REST API whenever a direct wireless connection is available. The data stored through the App Engine's Data store feature is then visualized through a J2EE web application which also allows users to manage data points from the sensor data. The second [2] paper proposes cloud integration into the field of Telemedicine. Remote EEG monitoring, or TeleEEG, surgery through remotely controlled robotic arms, medical record viewing, prescription monitoring and appointment scheduling are stated to already be in practice by surgeons and physicians. The paper adds to this by suggesting remote patient monitoring and patient admission through cloud services. Relieving the processing load from mobile devices to the server via the cloud is also proposed, which no doubt would give an edge over the necessity of heavy duty functional requirements such as signal pattern recognition. The third [3] implements a "Health Tracker 2000" which is composed of a temperature sensor, pulse sensor and an oximeter. This wearable device uses RFID tags to identify the patients and then the parameters monitored is then wirelessly relayed to a computer using the MICA2 and MICA2DOT components as the transceivers and receivers. The advantage of using such a system is its focus on a well-defined network organization that enables remote monitoring of the patient's data. This gave us the motivation to create our own method of communication between the base monitoring system and the information recipient and also to add an improvement to the portability factor by incorporating smart phone devices. The fourth [4] base paper utilizes ZigBee to communicate the electrocardiograph (ECG) information to two mobile embedded platforms using the HBE-ZigbeX. The ECG sensor first sends the ECG signal to a mobile embedded platform that classifies the received signal by the application of autoassociative multilayered perceptron networks after converting the raw ECG signal to a histogram of gradient form. The results are then sent to a second mobile embedded platform that acts an ECG state monitoring device. The usage of the histogram of gradient as the input for a multi-layered perceptron network has been implemented on the front end android device to detect abnormal signal readings and to alert the user about them. The final base paper [5] focuses on the design of a wearable heart rate, blood pressure and temperature sensor. The temperature is sensed by using a $10 \mathrm{~K}$ Ohms thermistor in a Wheatstone Bridge, the heart rate is measured by an ECG circuit and the blood pressure is calculated from the Pulse Width Transit Time which is the time interval between the peak of the ECG graph and the minimum trough of the photoplethysmograph (PPG) signal obtained by using a red LED. The signals received are conditioned through band pass filters and amplifiers and then wirelessly 
transferred to the user's phone. It is from this paper that we obtained the information we needed to design our own circuits for the modules proposed.

\section{Proposed Method}

The implementation requires several biomedical signal sensors to obtain parameters such as pulse rate, perspiration levels, and body temperature and oxygenation saturation. These parameters have been measured by implementing sensor circuits in combination with a microcontroller. The integration of these circuits to the medical frame would require "sensory arms" that would protrude toward the user at appropriate locations to acquire the necessary signals.

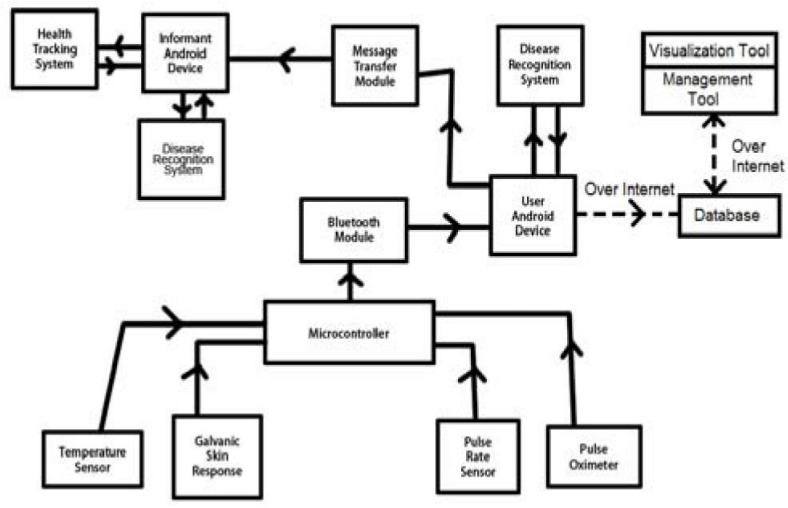

Figure 2 Proposed architecture

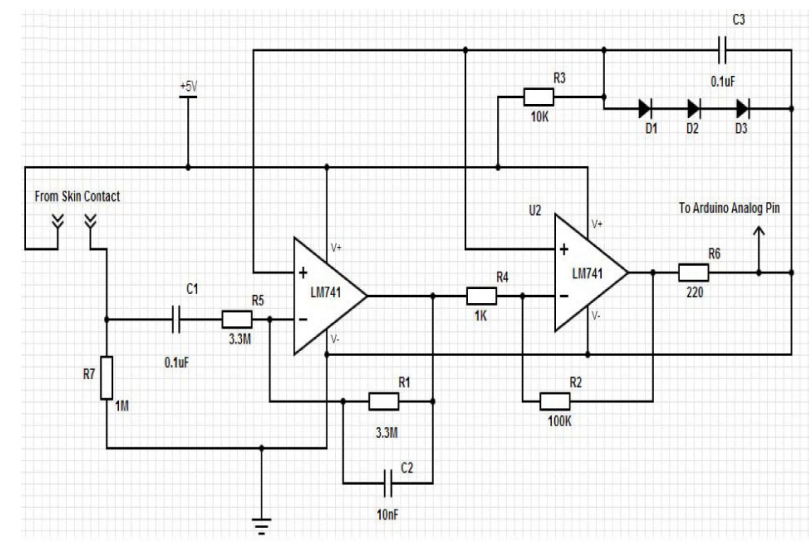

Figure 3 Circuit design for galvanic skin response sensor

The medical frame would also require a degree of computer assistance for the patient to understand the method of utilizing the medical frame. The method of wirelessly relaying the processed information from the medical frame to the doctor has been derived from [3] and [4]. The ability to send patient information over the cloud also allows a system of in-house treat- ment of the patient remotely from the doctor's office. Hence the complete virtualization of the treatment process is possible in cases where the ailment is one that is easy to diagnose, i.e. not one that has irregular symptoms. This also enables an international treatment system for countries with poor health care systems that have a deficit of doctors. The proposed architecture of implementation, shown in Figure 2, defines the final acceptor of the monitored parameters as a separate individual responsible for diagnostics, for example a doctor. The option of accessing the diagnostics over the internet has also been depicted to provide visualization and patient data management for concerned medical institutions. The method of operation via cloud services has been elucidated in the design and implementation section. The architecture can also be modified to make the final informant as a patient in order to allow self-monitoring and selfevaluation for those who wish to have on demand access to their vital signs status. The smart phone devices are equipped with an application that recognizes symptoms to deduce possible diseases and a tracking system that provides a visual display and user-friendly GUI of the parameters monitored.

\section{Design and Implementation}

The Arduino ATmega1280 has been selected as the microcontroller for the implementation. The sweat levels are detected through skin conductance using a Galvanic Skin Response Device. The galvanic metal used in skin contact is an aluminum foil attached to a piece of Velcro intended to be wrapped around the patient's finger as seen in Figure 4. The signal from the skin contact is passed through a high pass filter, low pass filter and then amplified as shown in Figure 3. The signals fluctuate according to the surface area perspiration percentage around the portion of the finger exposed to the foil. The signal received is then processed to obtain the perspiration percentage, which is then used to provide an approximation of sweat levels in the form of liters per day. The final implementation of the GSR sensor based on the circuit schematic can be seen in Figure 5. The body temperature of the patient is detected using the 10D-9 thermistor as the temperature sensor. The thermistor's resistance at $25 \mathrm{KC}$ is $10 \mathrm{Ohms}$ and decreases according to the increase in temperature. The thermistor circuit works on the principle of voltage dividing as shown in Figure 6 and whose implementation can be seen in Figure 7. The formula applied in Equation 1 on the voltage signal received obtains the resistance of the thermistor. 


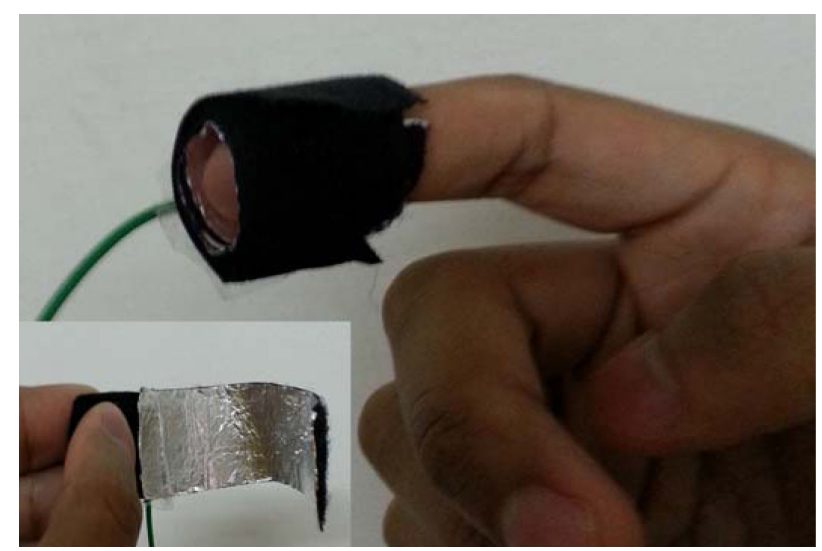

Figure 4 Galvanic skin contact

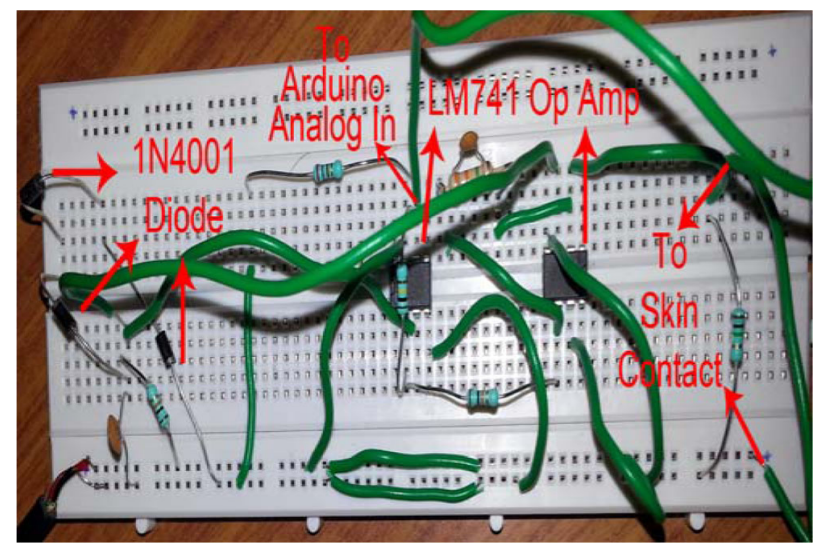

Figure 5 Implementation of GSR sensor

The voltage source of $5 \mathrm{~V}$ is used and the potentiometer is cranked to $1 \mathrm{~K} \mathrm{Ohms} \mathrm{resistance.} \mathrm{The} \mathrm{resistance} \mathrm{is}$ then applied to Equation 2 to obtain the Celsius reading of the thermistor. The Steinhart-Hart coefficient of the 10D-9 NTC thermistor is substituted to obtain Equation 2.

$$
\mathrm{R}=(\mathrm{S} V * \mathrm{P} R / S V-V),
$$

where $\mathrm{SV}$ is the source voltage, $\mathrm{PR}$ is the resistance the potentiometer is set to, and $\mathrm{V}$ is the voltage reading obtained from the raw analog input.

$$
\begin{array}{r}
\text { Temperature }=[(\log (R / 10) / 3000)+ \\
\left.(25+273.15)^{-1}\right]^{-1}-273.15
\end{array}
$$

The pulse oximeter and pulse rate sensors are based on a fundamental design from [5] and is intended to be used a shell that wraps around a thin part of the patient's body, such as the earlobe or a finger. Each shell encapsulates an LED and a photodiode to measure two different readings. The pulse rate is calculated by

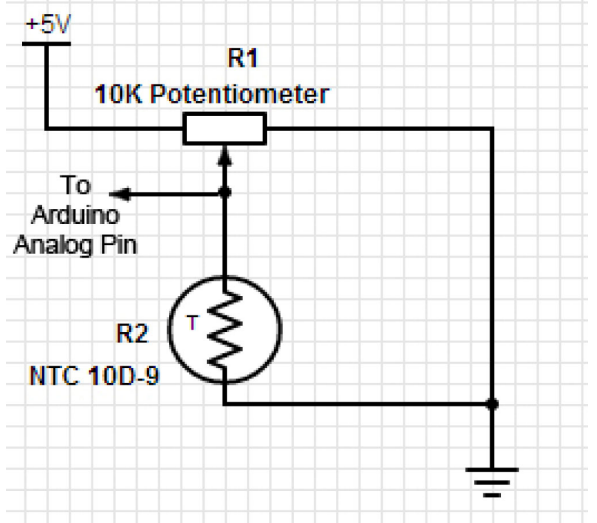

Figure 6 Circuit design for body temperature sensor

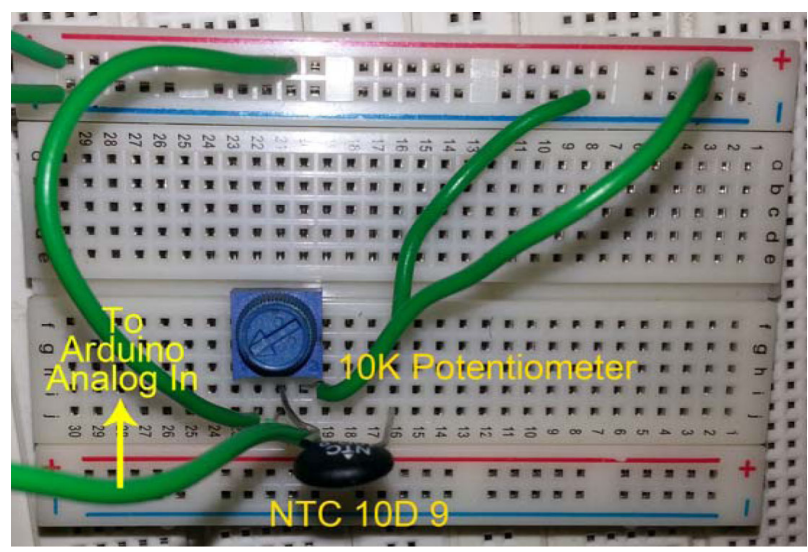

Figure 7 Implementation of body temperature sensor

using an infra-red LED in the photoplethysmograph circuit as shown in Figure 9, and a red LED is used to detect the ratio of oxygenated blood, which absorbs the red light, versus de-oxygenated blood, which absorbs infra-red light. The implementation of the circuit design can be seen in Figure 8. The photodiode used is the BPW34 due to its range of spectral responsivitiy which detects wavelengths between $700 \mathrm{~nm}$ to $600 \mathrm{~nm}$ with excellent sensitivity. The pulse rate is calculated by obtaining the total time taken obtain 10 peaks which is then scaled to obtain the number of beats per minute. The oxygen saturation percentage is obtained by tracking the ratio of the peak values. All measurements are automated by a microcontroller and are transferred to a smart phone device, in this case, and Android device, via the Bluetooth module HC-05 in order to implement a user friendly tracking system. Bluetooth has been selected over ZigBee, as suggested in [4], under the assumption that the user receiving the real time feedback lies within close proximity to the system. The HC-05 can be configured either as a master or a slave and the TX, RX, 3.3V and 


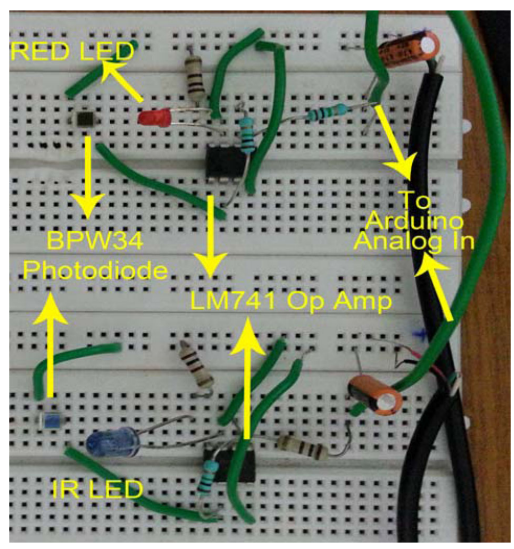

Figure 8 Implementation of oximeter

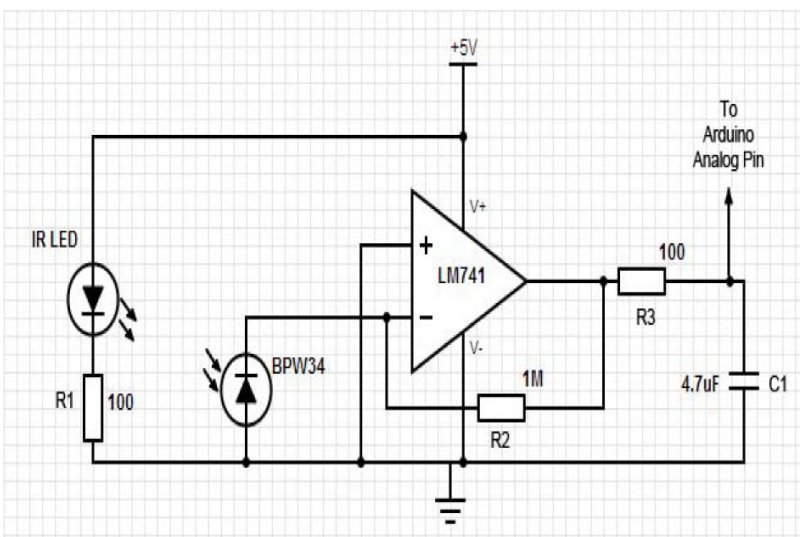

Figure 9 Circuit design for the photoplethysmograph sensor

GND pins are the only pins used in the implementation. The TX and RX pins are used for the serial transfer of data and commands to perform operations such as pairing and sending data. Due to the asynchronous manner of the Bluetooth module, the numerical data sent from the microcontroller is encoded. Cloud integration can be achieved by incrementing on the existing architecture with Figure 11. The figure illustrates the smart phone device communicating to the cloud by making a web service request. The smart phone must initially create a snapshot of data points which is uploaded to the server's database. The web service request executes a server side script that prepares the user's existing data snapshot to accommodate the snapshot sent by the smart phone. The request also executes a server side script that detects abnormal signals. If an abnormal signal is detected, a warning notification is pushed to the user's caregivers via email or SMS. The proposed cloud service provider is the Google App Engine which is a Platform As A Service platform that also has Software As A Service(SaaS) capabilities. The SaaS allows the development of a

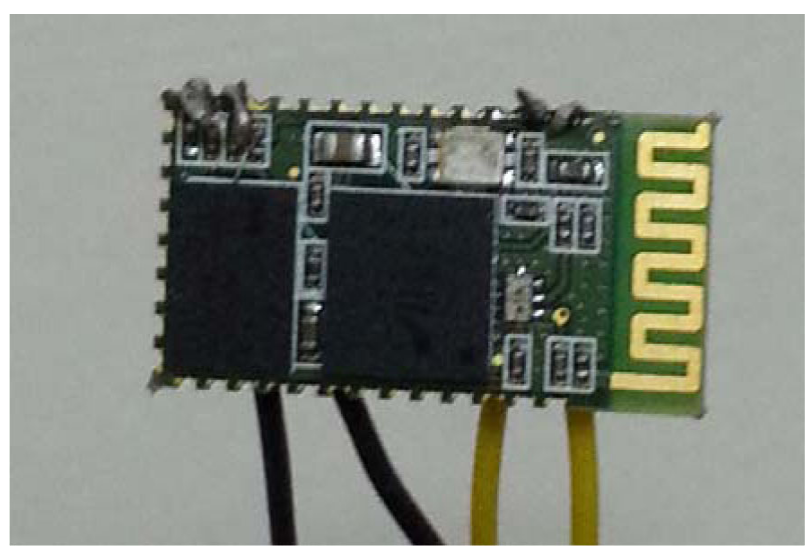

Figure 10 Snapshot of the HC-05 bluetooth module

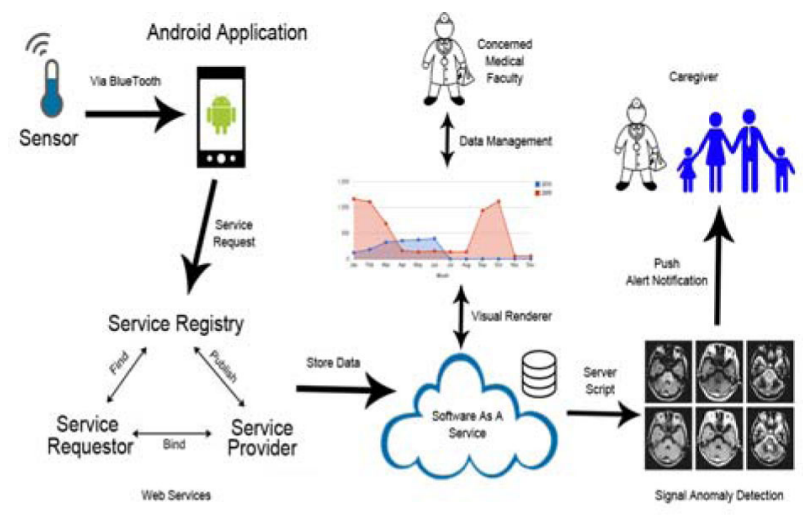

Figure 11 Cloud integration architectural design proposal

visual rendering and data management application of the snapshots obtained thus far. The algorithm of the code executed on the hardware is illustrated in Algorithm 1 .

\subsection{Algorithm 1 signal acquisition and transmit- ting}

1. procedure AcquireAndTransmit

2. BluetoothModule b;

3. Number analogR, celsius, analogPulse, analogPulse2, bpm, perspiration;

4. analogR $\longleftarrow$ ReadAnalog(TemperaturePin);

5. celsius $\longleftarrow$ caculateTemperature(analogR);

6. analogPulse $\longleftarrow$ ReadAnalog(PulsePin);

7. bpm « timeFor10Troughs(analogPulse);

8. analogPulse $2 \longleftarrow$ ReadAnalog(RedLEDPulsePin);

9. perspiration $\longleftarrow$ getOxygenation (analogPulse, analogPulse2); 
10. b.encodeAndSend(celsius,bpm,perspiration)

11. end procedure

The $n$-component- $n$ hardware details are listed as such:

1. Resistors
(a) $100 \mathrm{Ohm} \times 3$
(b) $1 \mathrm{M} \mathrm{Ohm} \times 4$
(c) $3.3 \mathrm{M} \mathrm{Ohm} \times 1$
(d) $100 \mathrm{~K} \mathrm{Ohm} \times 1$
(e) $10 \mathrm{~K} \mathrm{Ohm} \times 2$
(f) $220 \mathrm{Ohm} \times 1$
(g) $10 \mathrm{~K}$ potentiometer $\times 1$
(h) NTC thermistor $10 \mathrm{~d}-9$

2. Diodes

(a) IR Led 940mn wavelength $\times 1$

(b) Red Led $620 \mathrm{~nm}$ wavelength $\times 1$

(c) BPW34 Photodiode $\times 2$

(d) IN4001 Diode $\times 3$

3. Capacitors
(a) $4.7 \mu \mathrm{F} \times 2$
(b) $0.1 \mu \mathrm{F} \times 1$
(c) $10 v \mathrm{~F} \times 1$

\section{LM741 Op Amps $\times 4$}

5. Breadboard $\times 3$

6. AA Battery $\times 16$

\section{7. $20 \mathrm{~cm}$ Velcro Tape}

8. $6 \mathrm{~m}$ insulated copper wire

A disease recognition application was developed to allow a quick lookup of diseases according to the symptoms determined as seen in Figure 13 and Figure 14. A health tracking application was also developed to display the data received from the microcontroller, from Figure 15, which could also be displayed in the form of a real-time graphical representation [6] as seen in Figure 16-19. The operation of the application developed is illustrated in the control flow diagram in Figure 12. An assumption made is the diagnostic capability of the software portion of the implementation which should be well versed with the basic medical symptom evaluation techniques. The next assumption is that the records for all symptom/ailment details are enlisted in the database for the reference of the doctor.

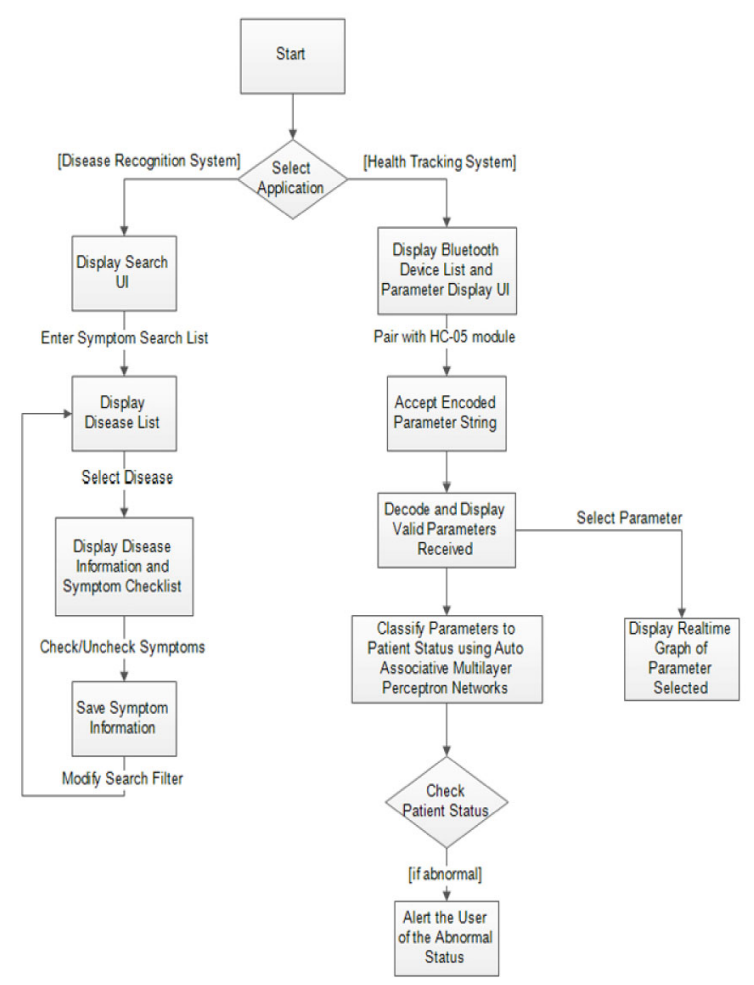

Figure 12 Control flow of the front end application

\section{Results}

The snapshot of the total system can be seen in Figure 20. One of the challenges faced by the implementation of the Bluetooth module was to ensure an acceptable rate of obtaining correct parameter strings on the smartphone device, and this was achieved by implementing an encoding scheme that contained a customized parity checking mechanism that allowed data retrieval through error correction. The performance throughput of the back-end has been recorded up to 200 stable detections per second, while the perfor-

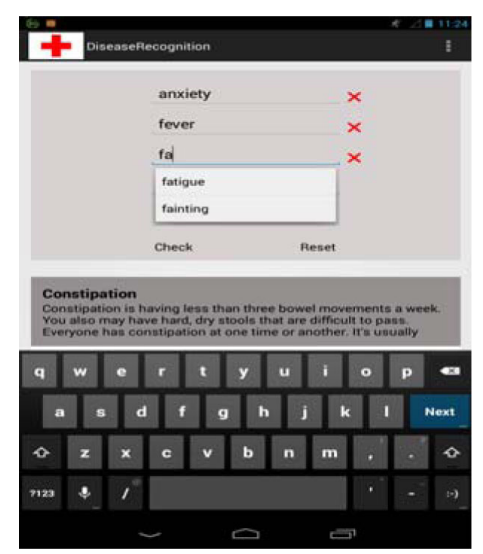

Figure 13 User interface for the disease recognition search function 


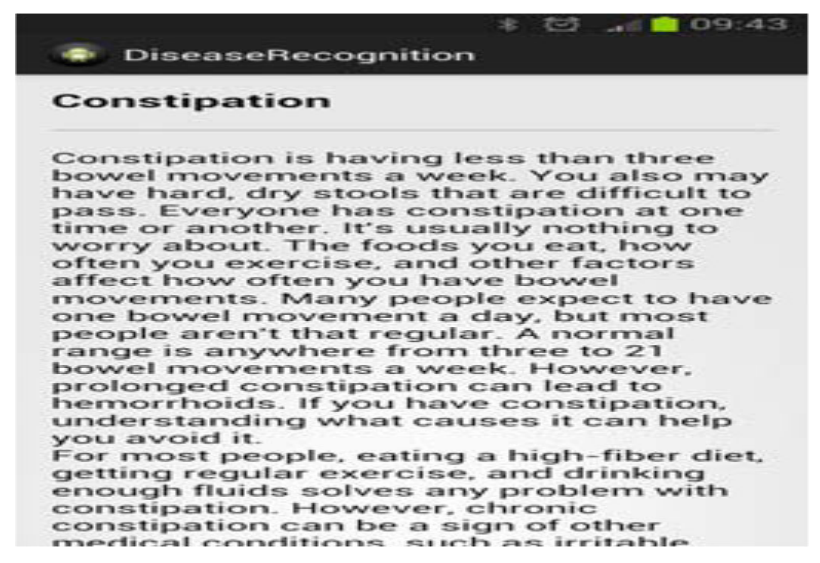

Figure 14 User interface for viewing the disease information

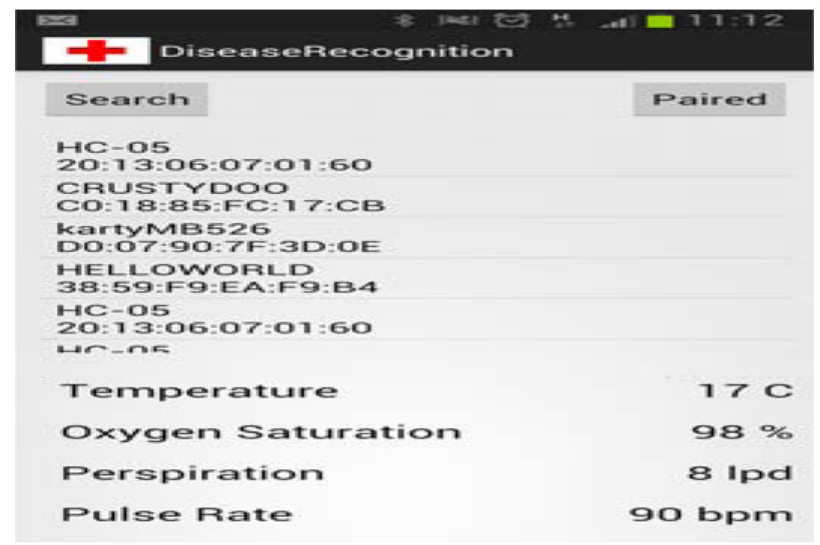

Figure 15 User interface for the health tracking system

mance throughput of data between the hardware to the software display has been recorded up to 50 detections per second of which $10 \%$ of the sent data was left unparsed since they corrupted data were filtered out in the decoding process. The testing phase of our project entailed an accuracy check of the parameters recorded with some monitoring tools purchased from the local pharmacy. The accuracy of the readings was initially not as high as expected and several modifications were performed on both the hardware and program ends to improve the final accuracy of the readings.

\section{Conclusion and Future Work}

The accuracy of the values received by the sensors used was high enough to obtain vital information about the state of the user. The back end hardware implementation allowed a continuous analysis of the user's vital signs with minimal processing latency. The scope of improvement in the implementation lies in the optimization of power consumption and a smaller circuit by converting the separate Photoplethysmograph and

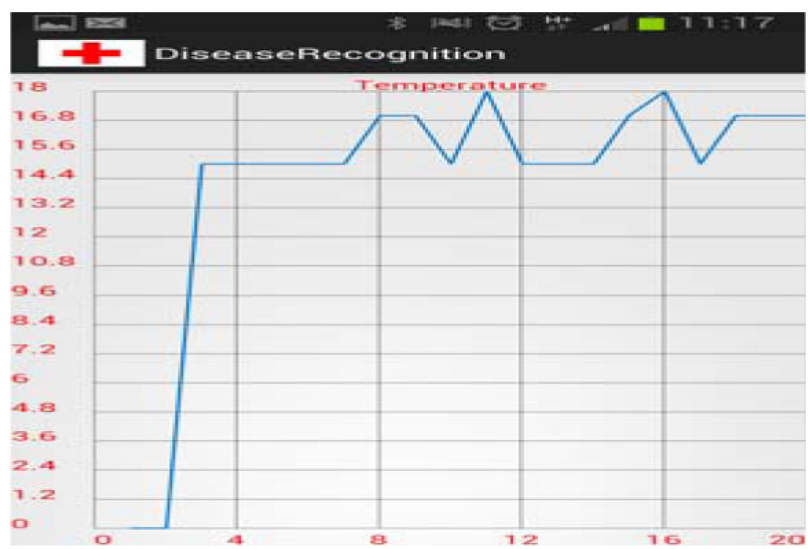

Figure 16 Real-time graphical representation of temperature from the health tracking system

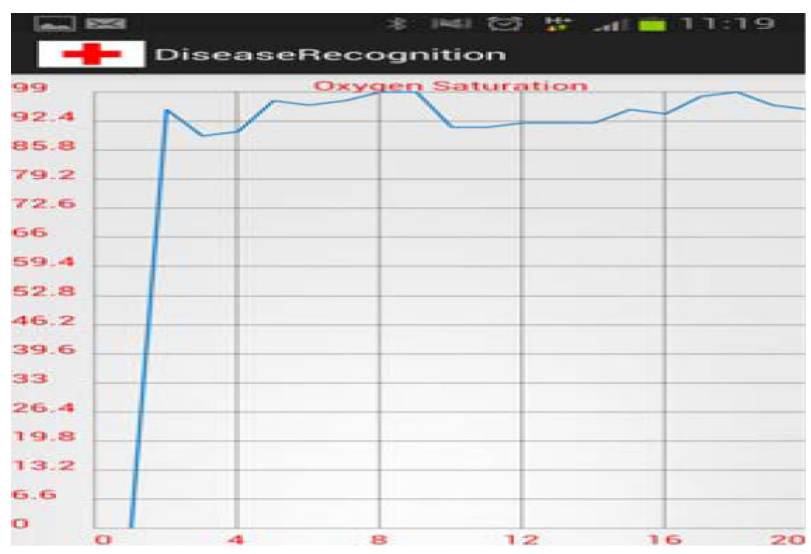

Figure 17 Real-time graphical representation of oxygen saturation from the health Tracking system

the Pulse Oximeter circuits into a single power source circuit that switches functionality using a multiplexer. Another improvement is to design an ergonomic shell that contains the photodiode and the LEDs. To achieve a closer simulation to the proposed Medical Frame, an intuitively designed mechanical set-up is required that scans the patients vital signs. The implementation only scans some of the several parameters required by the doctors and hence is open to addition of other scanners to obtain any necessary information about the patient. Carrying the project further, the integration of cloud services is yet to be implemented. The goal of this objective would be to successfully combine the health tracking application with cloud capabilities by uploading snapshot data of a fixed set of data points. This would also allow us to integrate processor intensive functionalities such as signal anomaly detection, which would be beneficial in the cases where the user is incapacitated and requires dire medical assistance. 


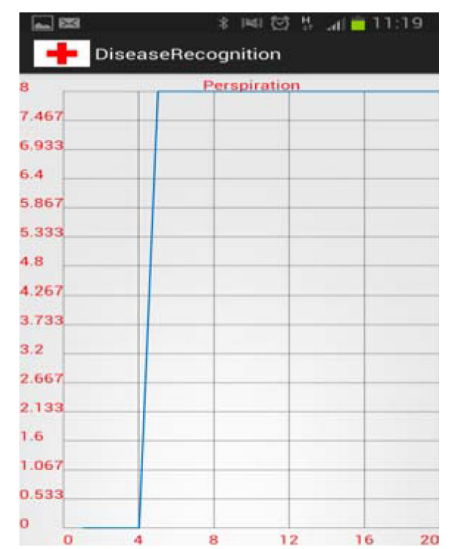

Figure 18 Real-time graphical representation of perspiration from the health tracking system

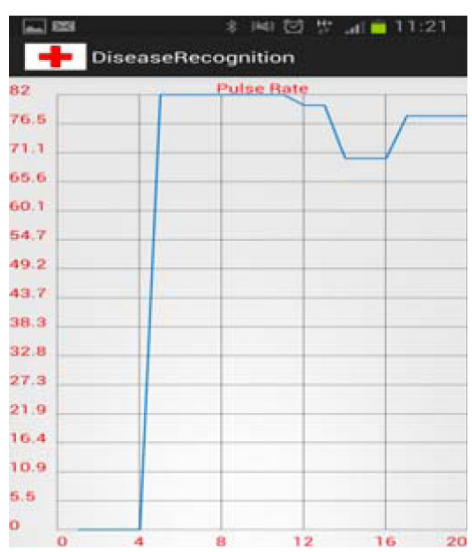

Figure 19 Real-time graphical representation of pulse rate from the health tracking system

\section{References}

[1] Charalampos Doukas, Ilias Maglogiannis, "Managing Wearable Sensor Data through Cloud Computing," IEEE Third International Conference on Cloud Computing Technology and Science, 2011, pp. 440445

[2] Princy Matlani, Narendra D Londhe, "A Cloud Computing Based Telemedicine Service," IEEE Point-ofCare Healthcare Technologies (PHT), 2013, pp. 326330

[3] Edward Teaw, Guofeng Hou, Michael Gouzman, K. Wendy Tang, Amy Kesluk, Matthew Kane and Jason Farrell, "A Wireless Health Monitoring System," IEEE International Conference on Information Acquisition, 2005.

[4] Hye-Jin Lee, Dong-Oh Kim, Bub-Joo Kang, SangWoo Ban, "Mobile Embedded Health-Care System Working on Wireless Sensor Network," Third International Conference on Communications and Mobile Computing, 2011, pp. 161-164

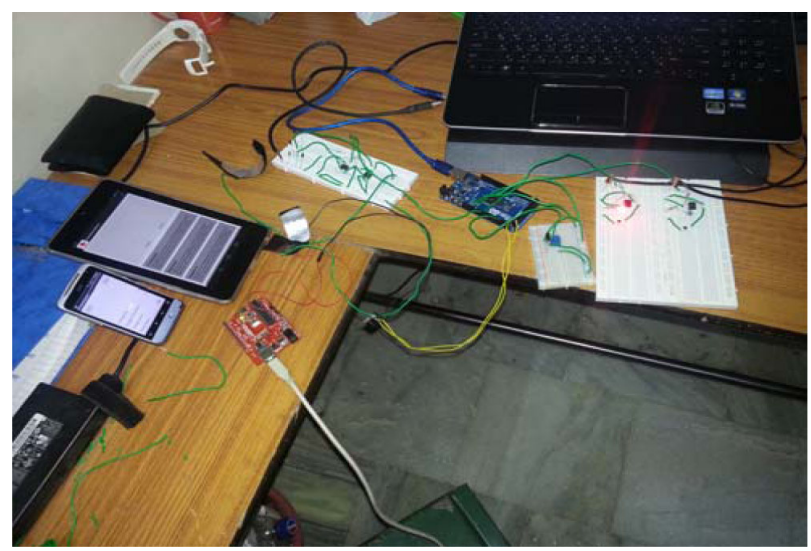

Figure 20 Snapshot of the final implementation

[5] Dhvani Parekh, "Designing Heart Rate, Blood Pressure and Body Temperature Sensors for Mobile OnCall System" (2010), EE 4BI6 Electrical Engineering Biomedical Capstones. Paper 39.

[6] Jonas Gehring, "Android GraphView," [Online]. Available: http://android-graphview.org 